\title{
Electron capture by finite-size polarizable molecules and clusters
}

\author{
E. E. Nikitin ${ }^{a b}$ and J. Troe ${ }^{* a c}$ \\ Received 22nd January 2010, Accepted 28th April 2010 \\ DOI: $10.1039 / \mathbf{c 0 0 1 5 1 9 a}$
}

\begin{abstract}
The effects of finite molecular target size are investigated for partial-wave selected capture of electrons by isotropically polarizable molecules and clusters within a generalized Vogt-Wannier model. It is shown how expressions for partial-wave selected capture probabilities of zero-size targets from Dashevskaya et al. (Phys. Chem. Chem. Phys., 2009, 11, 9364) can be modified to account for finite target sizes of the molecules and clusters. The transition from quantum to classical, from single- to multiple- and all-wave, behaviour of capture probabilities, cross sections, and rate constants is illustrated.
\end{abstract}

\section{Introduction}

Electron attachment to polarizable neutral molecules is often treated in terms of the Vogt-Wannier model for electron capture $^{1-4}$ in an interaction potential

$$
V(r)=-\mathrm{e}^{2} \alpha / 2 r^{4}
$$

where $\alpha$ is the polarizability of the neutral molecule and $r$ is the center-of-mass distance between the molecule and the electron. Obviously, this potential is realistic only for large $r$. The accommodation of the incoming electron by the molecular electrons and the nuclear frame, through "electron-phonon coupling" or "intramolecular vibrational redistribution (IVR)", at small $r$ requires a different treatment without separation of electronic and nuclear coordinates, e.g. in terms of $R$-matrix theory. ${ }^{2,4}$ Nevertheless, Vogt-Wannier electron capture theory provides a most useful reference for comparison with experimental data. Electron attachment by $\mathrm{SF}_{6}$ at low electron energies, for instance, is well characterized ${ }^{2,4,5}$ by s-wave capture and the Vogt-Wannier relationships, which indicate that the long-range part of the potential of eqn (1.1) here is adequate enough. With increasing energy, however, the IVR problem becomes more serious and eqn (1.1) cannot be considered sufficient for short ranges. In ref. 5, the deviations from the Vogt-Wannier relationships were empirically represented by an "IVR-factor" smaller than unity, introduced to quantitatively characterize the deviations.

When the size of the molecular species increases, such as in electron attachment to clusters or nanoparticles, the shortrange problem with eqn (1.1) becomes even more serious. In this case, the finite size of the electron-attaching target often is treated on the basis of a potential

$$
V(r)=-\mathrm{e}^{2} \alpha /\left[2 r^{2}\left(r^{2}-r_{0}^{2}\right)\right]
$$

where $r_{0}$ denotes the radius of the target species and, for the particular model of a conducting sphere, $r_{0}$ is simply related to

\footnotetext{
${ }^{a}$ Max-Planck-Institut für Biophysikalische Chemie, Am Fassberg 11, D-37077 Göttingen, Germany. E-mail: shoff@gwdg.de

${ }^{b}$ Schulich Faculty of Chemistry, Technion-Israel Institute of

Technology, Haifa 3200, Israel

${ }^{c}$ Institut für Physikalische Chemie, Universität Göttingen,

Tammannstrasse 6, D-37077 Göttingen, Germany
}

the polarizability by ${ }^{6} r_{0}{ }^{3} \approx \alpha$. Adding centrifugal energy of the orbital motion and determining the centrifugal maxima of the effective potential, the classical capture cross section $\sigma_{\text {cap }}(E)$ for the potential of eqn (1.2) takes the simple form ${ }^{7}$

$$
\sigma_{\text {cap }}(E)=\pi r_{0}^{2}+\left(2 \pi^{2} \alpha \mathrm{e}^{2} / E\right)^{1 / 2}
$$

where the first term is the geometrical target cross section and the second term is the classical Langevin cross section for a zero-size polarizable target. After thermal averaging, eqn (1.3) leads to the thermal capture rate constant

$$
k_{\text {cap }}(T)=\pi r_{0}^{2}\left(8 k_{\mathrm{B}} T / \pi \mu\right)^{1 / 2}+k_{L}
$$

with the Langevin rate constant

$$
k_{L}=2 \pi \mathrm{e}(\alpha / \mu)^{1 / 2}
$$

Eqn (1.3) is relevant for electron capture by large clusters and nanoparticles under classical conditions, see the reviews of ref. 4, 8 and 9. To mention two specific examples, eqn (1.3) in ref. 10 was used for the analysis of cross sections of collisions between electrons and sodium nanoclusters $\mathrm{Na}_{9000}$ with $r_{0} \approx 4 \mathrm{~nm}$; likewise, eqn (1.3) in ref. 11 was applied to the much smaller cluster $\mathrm{C}_{60}$, for which $r_{0}=0.35 \mathrm{~nm} .^{12}$

The classical eqn (1.3) and (1.4) require that many partial waves of the electronic motion contribute to the capture process, that the centrifugal barriers are located at long range with $r \gg r_{0}$, and that the energies are such that tunnelling through the barriers is of minor importance. There is, however, also an interest in intermediate situations, where only a limited number of partial waves contribute. For instance, for electron attachment to $\mathrm{C}_{60}$ the question arises ${ }^{13}$ whether the finite size of $\mathrm{C}_{60}$ still can be described by the classical eqn (1.3) such as this was done in ref. 11 . This intermediate range is the issue of the present article. We first extend the analytical representation of Vogt-Wannier capture probabilities for zero-size targets from earlier work (up to $l=4$, see ref. 2 and 13) up to $l=12$. Then we extend the Vogt-Wannier relationships to finite-size targets for partial waves where there are centrifugal barriers (i.e., for $l \geq 1$ ). We conclude that the size effects diminish with decreasing $l$, while they approach the results of the classical eqn (1.3) at large $l$. 


\section{Finite-size effects in capture probabilities}

We represent the capture cross section $\sigma_{\text {cap }}(k)$, with the wave vector of the relative motion $k=p / \hbar=\sqrt{2 \mu E / \hbar}$, as a sum of partial-wave contributions

$$
\sigma_{\text {cap }}=\sum_{l} \sigma_{\text {cap }, l}(k)=\sum_{l}\left(\pi / k^{2}\right)(2 l+1) P_{l}(k)
$$

where the $P_{l}(k)$ denote the $l$-specific transmission coefficients of partial waves across the effective potential $V_{\text {eff }}(r, l)$. The latter is given by

$$
V_{\mathrm{eff}}(r, l)=V(r)+l(l+1) \hbar^{2} / 2 \mu r^{2}
$$

with $V(r)$ e.g. from eqn (1.2). We use dimensionless distances, $\rho=r / R_{s}$ and $\rho_{0}=r_{0} / R_{s}$, and wave vectors, $\kappa=k R_{s}$, with the scaling parameter $R_{s}=\left(\alpha \mu \mathrm{e}^{2} / \hbar^{2}\right)^{1 / 2}$, as well as dimensionless energies, $\varepsilon=E / E_{s}$ and $v_{\text {eff }}(\rho)=V_{\text {eff }}(r, l) / E_{s}$, with the scaling parameter $E_{s}=\hbar^{4} / \alpha \mu^{2} \mathrm{e}^{2}$, see ref. 3. The corresponding Schrödinger equation has the form

$$
\frac{1}{2} \frac{\mathrm{d}^{2} \psi(\rho)}{\mathrm{d} \rho^{2}}+v_{\mathrm{eff}}(\rho) \psi(\rho)=\frac{\kappa^{2}}{2} \psi(\rho)
$$

where

$$
v_{\mathrm{eff}}(\rho, l)=l(l+1) / 2 \rho^{2}-1 /\left[2 \rho^{2}\left(\rho^{2}-\rho_{0}^{2}\right)\right]
$$

With the proper capture boundary condition, the solution of eqn (2.3) leads to transmission coefficients, representing the capture probabilities, such as demonstrated for zero-size targets $\left(\rho_{0}=0\right)$ in ref. 14 and 15 . As we will express finite-size capture probabilities relative to the corresponding zero-size quantities, we present the latter here again such as derived in ref. 15. For s-waves $(l=0)$, our optimum analytical approximation was expressed in the form

$$
P_{l=0}(\kappa) \approx 1-0.25 \exp (-1.41 \kappa)-0.75 \exp (-4.86 \kappa)
$$

For higher waves $(l>0)$, the following expressions were found to perform very well:

$$
P_{l>0}(\kappa) \approx \begin{cases}\left(\kappa / \kappa_{l}^{*}\right)^{2 l+1} g_{l}\left(\kappa_{l}^{*}\right) & \text { for } \kappa<\kappa_{l}^{*} \\ g_{l}(\kappa) & \text { for } \kappa \geq \kappa_{l}^{*}\end{cases}
$$

where

$$
g_{l}(\kappa)=1 /\left\{1+\exp \left[-2 H_{l}(\kappa)\right]\right\}
$$

and

$$
H_{1}(\kappa)=a_{1}\left(\kappa-\kappa_{1}^{(1 / 2)}\right)+b_{1}\left(\kappa-\kappa_{1}^{(1 / 2)}\right)+c_{1}\left(\kappa-\kappa_{1}^{(1 / 2)}\right)^{3}
$$

The $l$-specific parameters $a_{l}, b_{l}, c_{l}, \kappa_{l}^{(1 / 2)}$, and $\kappa_{l}{ }^{*}$ derived are summarized in Table 1 for $l=1-12$, extending our earlier results for $l=1-4$ from ref. 14 and 15. Eqn (2.5), for s-waves, was shown to be precise within better than 1 percent. Eqn (2.6)-(2.8) with the parameters of Table 1, on the other hand, were found to be accurate within a few percent such as documented for $l=1-4$ in ref. 15 .

While the Schrödinger eqn (2.3) could be solved easily for the zero-target size Vogt-Wannier model, the particular short-range form of the potential of eqn (1.2) created problems for finite target sizes, i.e. for $\rho_{0} \neq 0$. However, since the potential of eqn (1.2) is artificial anyway and does not account for the
Table 1 Parameters in eqn (2.6)-(2.8) for capture probabilities of electrons by zero-size targets $\left(\rho_{0}=0\right.$; results for $l=1-4$ from ref. 14 and 15)

\begin{tabular}{lrrlll}
\hline$l$ & $\kappa_{l}^{(1 / 2)}$ & $\kappa_{l}^{*}$ & $a_{l}$ & $b_{l}$ & $c_{l}$ \\
\hline 1 & 1.187 & 0.68 & 1.50 & -0.50 & 0.10 \\
2 & 3.186 & 1.64 & 0.870 & -0.150 & $3.00 \times 10^{-2}$ \\
3 & 6.186 & 2.55 & 0.610 & $-4.80 \times 10^{-2}$ & $9.50 \times 10^{-3}$ \\
4 & 10.186 & 4.95 & 0.485 & $-2.50 \times 10^{-2}$ & $1.70 \times 10^{-3}$ \\
5 & 15.186 & 7.00 & 0.400 & $-1.05 \times 10^{-2}$ & $1.05 \times 10^{-3}$ \\
6 & 21.186 & 12.5 & 0.340 & $-8.25 \times 10^{-3}$ & $2.00 \times 10^{-4}$ \\
7 & 28.186 & 17.5 & 0.300 & $-4.25 \times 10^{-3}$ & $1.25 \times 10^{-4}$ \\
8 & 36.186 & 22.0 & 0.260 & $-3.60 \times 10^{-3}$ & $5.00 \times 10^{-5}$ \\
9 & 45.186 & 29.0 & 0.230 & $-3.00 \times 10^{-3}$ & $1.25 \times 10^{-5}$ \\
10 & 55.186 & 36.0 & 0.207 & $-2.40 \times 10^{-3}$ & 0 \\
11 & 66.186 & 48.0 & 0.193 & $-1.54 \times 10^{-3}$ & 0 \\
12 & 78.186 & 55.0 & 0.180 & $-1.08 \times 10^{-3}$ & 0 \\
\hline
\end{tabular}

short-range IVR problem, we only considered this potential in the region of its centrifugal maxima (for $l \geq 1$ ). Here, we replaced the effective potential of eqn (2.4) by an inverted Morse (iM) potential and employed its transmission coefficients both for zero and finite target sizes, with the goal of obtaining a finite-target size generalization of the Vogt-Wannier capture model. This procedure clearly is limited to $l \geq 1$, where there are centrifugal barriers, and s-waves are not included in the treatment. Also, capture probabilities at small energies, deep in the tunnelling range, will not be represented properly. However, for large energies the approach is shown to work satisfactorily for the zero-target size Vogt-Wannier model, which makes the approach also suitable for an extension to finite target sizes. We provide this extension in the form of a

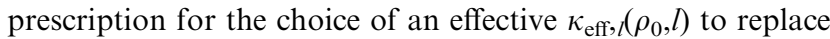
the dimensionless wave vector $\kappa$ (being proportional to $\sqrt{E}$ ) in eqn (2.6)-(2.8) such that finite-size effects are accounted for approximately. We emphasize that our procedure anticipates numerical results for more adequate short-range potentials. Nonetheless, its simplicity and reasonable physical foundations at this stage makes the approach useful for quick estimates of finite-target size effects of a variety of potentials.

For $l>0$, in our approach we approximate the effective potential $v_{\mathrm{eff}}(\rho)$ by an inverted Morse (iM) potential for which transmission coefficients are available in explicit analytical form (see ref. 16-20). The iM potential has the form

$$
v_{\mathrm{eff}}(\rho) / v_{\mathrm{eff}, \max }=\left\{1-\left[1-\exp \left(-\left(\rho-\rho_{\max }\right) / a\right)\right]^{2}\right\}
$$

where $v_{\mathrm{eff}, \max }$ is the maximum value of $v_{\mathrm{eff}}(\rho)$, being located at $\rho=\rho_{\max }$, and $a$ is a free fit parameter. The transmission coefficients then are given by ${ }^{19}$

$$
P_{l}^{\mathrm{iM}}(\kappa)=[1-\exp (-4 \pi f)] /[1+\exp \{2 \pi(q-f)]\}
$$

where $f^{2}=\kappa^{2} a^{2}$ and $q^{2}=2 v_{\text {eff, max }} a^{2}$. The probabilities $P_{l}^{\mathrm{iM}}$ from eqn (2.10) in our work are used to approximate the partial wave transmission probabilities $P_{l}$ for the effective potential $v_{\text {eff }}(\rho, l)$ of eqn (2.4).

For $l>0$, the potential of eqn (2.4) possesses a maximum, and the parameter $a$ can be determined by fitting the iM potential to this potential in such a way that the maxima $v_{\text {eff,max }}$ and the locations $\rho_{\text {max }}$ of the maxima for a given $l$ are correctly reproduced. This is achieved by putting

$$
\rho_{\max }=\left\{\left(z+z^{1 / 2}\right) /[1(l+1)]^{1 / 2}\right\}
$$


and

$$
v_{\text {eff, } \max }=\left\{[l(l+1)] /\left[1+z^{1 / 2}\right]\right\}^{2} / 2
$$

where $z=l(l+1) \rho_{0}^{2}+1$. In addition, the parameter $a$ should be fitted. We have done this in two ways: either the halfwidths $\Delta \rho_{1 / 2}$ or the second derivatives at maximum of the two potentials of eqn (2.4) and (2.9) were forced to be equal. In the latter case, one has

$$
\frac{\mathrm{d}^{2} v_{\mathrm{eff}}\left(\rho=\rho_{\max }, l\right)}{\mathrm{d} \rho^{2}}=-4\left(2 v_{\mathrm{eff}, \max }\right)^{3 / 2}
$$

such that

$$
a=\left(2 v_{\text {eff,max }}\right)^{-1 / 4} / 2
$$

As an example, for $l=2$ and $\rho_{0}=0.3$, the halfwidth-based value of $a$ has the value $a=0.382$, whereas the second derivative-based value of $a$ is equal to $a=0.306$, see the illustrations shown in the following.

In Fig. 1, we first demonstrate the influence of a finite target size on the effective potential of eqn (2.4) by putting $\rho_{0}=0.3$. Whereas hardly any influence is noticeable for $l=0$ where $v_{\text {eff,max }}=0$, the maximum $\nu_{\text {eff,max }}$ is reduced for $l=2$. For $l>0, v_{\mathrm{eff}, \max }$ and the width of the barrier with increasing $\rho_{0}$ are increasingly reduced. As a consequence, one expects increasing capture probabilities with increasing $\rho_{0}$.

Fig. 2, for $l=2$ and $\rho_{0}=0.3$, compares the effective potential of eqn (2.4) with the iM potential of eqn (2.9) after fitting of its parameters in the way described before. The fits look satisfactory but reproduce the potential of eqn (2.4) in different ways. The halfwidth-based fit (with $a=0.382$ ) works better for smaller energies whereas the second derivative-based fit (with $a=0.306$ ) looks better for larger energies. These differences translate into minor differences of the modelled transmission coefficients. For a number of reasons, we have preferred the latter fit, see below.

Before investigating the influence of finite target sizes on the transmission coefficients $P_{l}(\kappa)$, for zero-size targets we test the

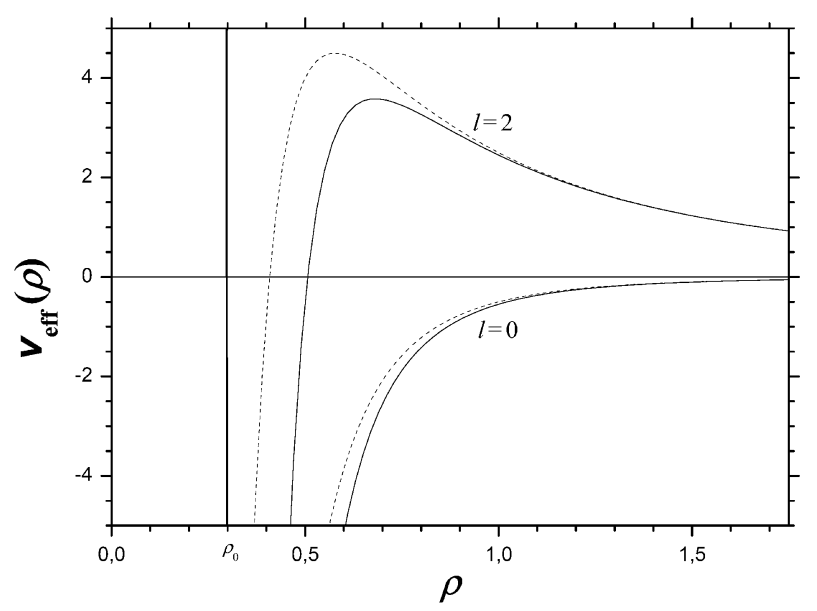

Fig. 1 Effective potentials $v_{\text {eff }}(\rho)$ of eqn (2.4) for $l=0$ and $l=2$ (dashed lines: zero-size target, $\rho_{0}=0$; full lines: finite-size target, $\left.\rho_{0}=0.3\right)$.

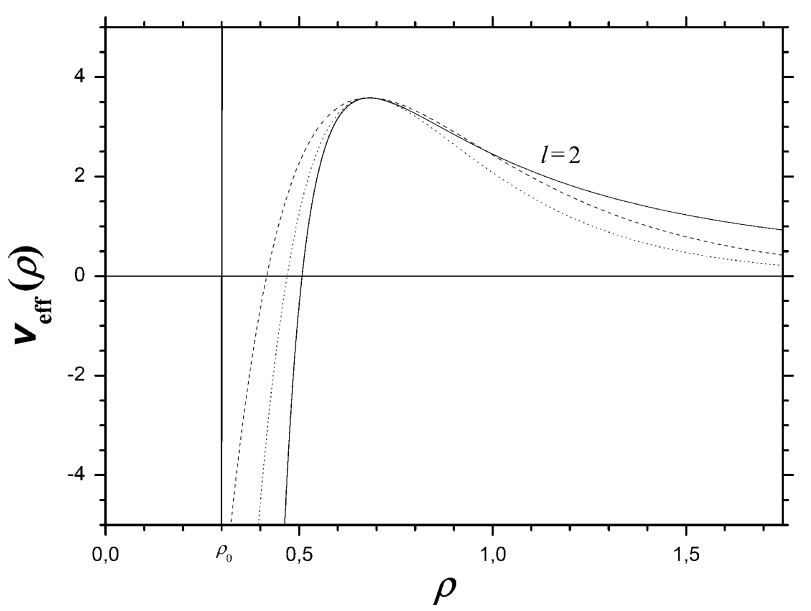

Fig. 2 Fit of effective potentials $v_{\text {eff }}(\rho)$ of eqn (2.4) for $l=2$ and $\rho_{0}=0.3$ by inverted Morse potentials iM of eqn (2.9) (full line: eqn (2.4), dashed line: halfwidth-based iM with $a=0.382$, dotted line: second derivative-based iM with $a=0.306$, see text).

consequences of using iM potentials instead of the polarization potential of eqn (1.1) (with $r_{0}=0$ ). This can only be done for $l$ larger than zero when maxima of the potentials arise. By comparing iM results with accurate results from ref. 3, Fig. 3 shows the results for $l=1-5$. For a better representation, instead of $\kappa$ an abscissa $\lambda=-1 / 2+[1 / 4+2 \kappa]^{1 / 2}$ is chosen where $\kappa=(2 \varepsilon)^{1 / 2}$, see ref. 3. We consider two versions of the iM results of eqn (2.10), the full iM result of eqn (2.10) and the simplified result (siM) omitting the exponential term in the numerator of eqn (2.10). Such as demonstrated for $l=1$ in Fig. 3 , the iM and siM results nearly agree for $\lambda$ larger than 0.5 and $P_{l}^{\mathrm{iM}}(\kappa)$ larger than about 0.2 . For $\lambda<0.5$, the iM, the $\mathrm{siM}$, and the accurate results increasingly disagree. This is not unexpected because the potentials differ in the tunnelling range where the transmission coefficients most sensitively depend on the potential. With increasing $l$, the iM and siM results agree increasingly better with the accurate results. Fig. 3 shows that, apart from the described differences, the iM and siM results for $l \geq 1$ quite well approximate the accurate transmission coefficients for the potential of eqn (1.1). In the following we expect that this will also be the case for finite target sizes. In order to improve the quality of the results, instead of using the $\mathrm{iM}$ and siM results directly, however, we only determine the shift of the transmission coefficient curves to the left for finite target sizes by the iM model and we apply the derived shift-relation to the accurate zero-size target transmission coefficients. Fig. 4 illustrates the effect for $P_{l}^{\mathrm{iM}}{ }_{2}\left(\kappa, \rho_{0}\right)$.

In the following we describe how a simple shift-relationship can be obtained. We ask how $\kappa$ in $P_{l}^{\mathrm{iM}}\left(\kappa, \rho_{0}=0\right)$ should be replaced by an effective value $\kappa_{\text {eff }}\left(\kappa, l, \rho_{0}\right)$ such that the general $P_{l}^{\mathrm{iM}}\left(\kappa, \rho_{0}\right)$ is recovered. We then assume that approximately the same shift-relationship can be used for the relation between the accurate $P_{l}\left(\kappa, \rho_{0}=0\right)$ and the general $P_{l}\left(\kappa, \rho_{0}\right)$. As the effects are small, we expect this approximation to hold sufficiently well in practical applications.

The desired shift-relation can be derived from eqn (2.10) by observing that, if the exponent in the numerator of the r.h.s. is large enough (e.g. for $\kappa>1 / 2, l>0,0<\rho_{0}<0.5$, and 


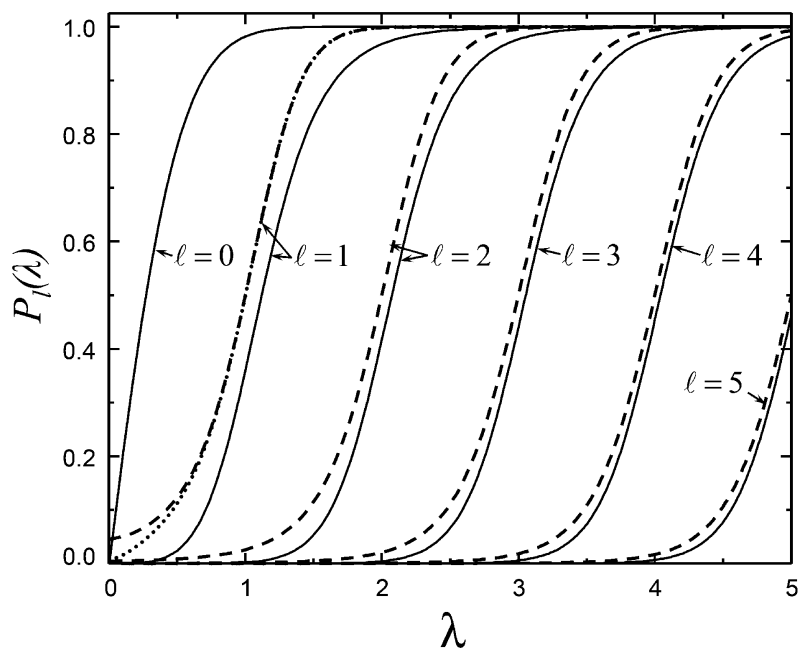

Fig. 3 Capture probabilities $P_{l}(\lambda)$ for zero-size targets $(\lambda=-1 / 2+$ $\left[1 / 4+2(2 \varepsilon)^{1 / 2}\right]^{1 / 2}, \varepsilon=$ scaled energy, see text; full lines: accurate results from eqn (2.5)-(2.8); dotted line for $l=1$ : results for inverted Morse (iM) potential from eqn (2.10); dashed lines: results for simplified inverted Morse (siM) potential from eqn (2.10) neglecting the exponential term in the numerator).

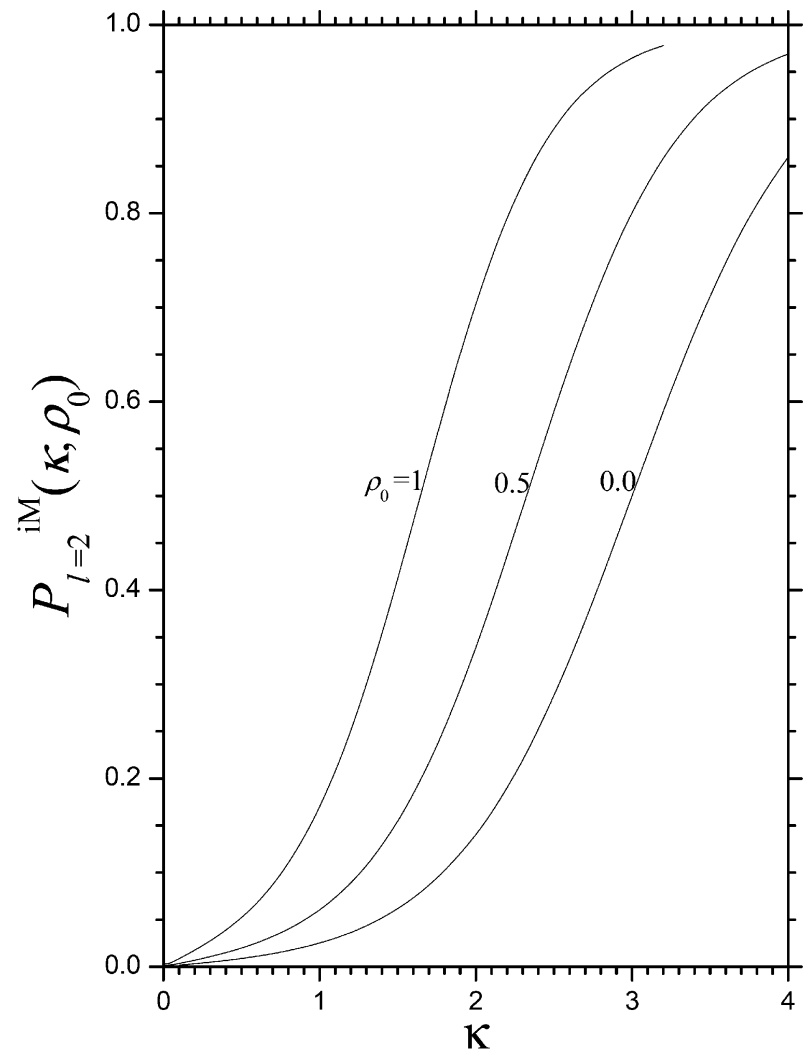

Fig. 4 Capture probabilities $P_{l=2}^{\mathrm{i} M}\left(\kappa, \rho_{0}\right)$ for inverted Morse potential and scaled target sizes $\rho_{0}=0,0.5$, and 1.0 .

$\left.4 \pi a\left(l, \rho_{0}\right) \kappa>\pi\right)$, the exponential term in the numerator can be ignored, and $P_{l}^{\mathrm{iM}}(\kappa)$ assumes the simplified form

$$
P_{1}^{\mathrm{siM}}\left(\kappa, \rho_{0}\right)=\frac{1}{1+\exp \left[2 \pi a\left(l, \rho_{0}\right)\left(\kappa_{\mathrm{th}}\left(l, \rho_{0}\right)-\kappa\right)\right]}
$$

where $\kappa_{\mathrm{th}}\left(l, \rho_{0}\right)$ is given by

$$
\kappa_{\mathrm{th}}\left(l, \rho_{0}\right)=\left(2 v_{\mathrm{eff}, \max }\right)^{1 / 2}
$$

This is the siM model illustrated in Fig. 3 before. As an interesting property of the $P_{l}^{\mathrm{siM}}\left(\kappa, \rho_{0}\right)$ one observes that any $P_{l}^{\mathrm{siM}}\left(\kappa, \rho_{0}\right)$ can be generated from $P_{l}^{\mathrm{siM}}(\kappa, 0)$ by a simple transformation $\kappa \rightarrow \kappa_{\text {eff }}\left(\kappa, l, \rho_{0}\right)$. This transformation is obtained by comparing $P_{l}^{\mathrm{siM}}\left(\kappa, \rho_{0}\right)$ and $P_{l}^{\mathrm{siM}}\left(\kappa_{\mathrm{eff}}, \rho_{0}=0\right)$. It leads to

$$
\kappa_{\text {eff }}\left(\kappa, l, \rho_{0}\right)=\frac{a\left(l, \rho_{0}\right)}{a\left(l, \rho_{0}=0\right)}\left(\kappa-\kappa_{\text {th }}\left(l, \rho_{0}\right)\right)+\kappa_{\text {th }}\left(l, \rho_{0}=0\right)
$$

With the help of eqn (2.12) and (2.13), eqn (2.17) can be rewritten as

$$
\kappa_{\mathrm{eff}}\left(\kappa, l, \rho_{0}\right)=B\left(l, \rho_{0}\right)\left(\kappa-\kappa_{\mathrm{th}}\left(l, \rho_{0}\right)\right)+\kappa_{\mathrm{th}}\left(l, \rho_{0}=0\right),
$$

with $B\left(l, \rho_{0}\right)$ and $\kappa_{\mathrm{th}}\left(l, \rho_{0}\right)$ explicitly given as

$$
B\left(l, \rho_{0}\right)=\left(\frac{1+\sqrt{l(l+1) \rho_{0}^{2}+1}}{2}\right)^{1 / 2}
$$

and

$$
\kappa_{\mathrm{th}}\left(l, \rho_{0}\right)=\frac{l(l+1)}{1+\sqrt{l(l+1) \rho_{0}^{2}+1}}
$$

This shift-relation leads to the correct classical limit, when the number of terms in eqn (2.1) is large.

A simpler shift relation, evaluated through the shifts of the $\kappa$-values for which $P_{l}^{\mathrm{iM}}\left(\kappa, \rho_{0}\right)=0.5$, reads

$$
\kappa_{\mathrm{eff}}\left(\rho_{0}, l\right) \approx \kappa\left(\rho_{0}=0, l\right)\left[1+0.37 l^{1.4} \rho_{0}{ }^{1.75}\right]
$$

It describes, at least qualitatively, the way in which $\kappa_{\text {eff }}$ increases with increasing $l$ and $\rho_{0}$. This shift-relation holds reasonably well, but it does not lead to the correct classical limit. The iM model is not restricted to integer $l$, but can also be generalized to noninteger $l$. For $l$ approaching zero, eqn (2.21) then suggests that $\kappa_{\text {eff }}\left(\rho_{0}, l \rightarrow 0\right) \approx \kappa\left(\rho_{0}=0, l \rightarrow 0\right)$, i.e. finite-size effects should disappear for s-waves. Similar conclusions are obtained when $\kappa_{\text {eff }}\left(\kappa, l, \rho_{0}\right)$ from eqn $(2.18)-(2.20)$ is considered. However, a rigorous confirmation of this conclusion remains to be given.

The performance of the recommended shift-relationship of eqn (2.18)-(2.20) is excellent over the range $0.1<P<1$. Increasingly larger deviations are observed only for smaller values of $P$, i.e. deeper in the tunnelling range. According to the calculations of this section, optimum results for capture probabilities with finite-size targets are obtained, if $\kappa$ in the expression for $P_{l}\left(\kappa, \rho_{0}=0\right)$ from ref. 15 , such as given by eqn (2.6)-(2.8), is replaced by $\kappa_{\text {eff }}\left(\rho_{0}, l\right)$ from eqn (2.18)-(2.21).

\section{Finite-size effects in capture cross sections and thermal capture rate constants}

Making use of the transmission coefficients $P_{l}\left(\kappa, \rho_{0}\right)$ elaborated in section 2, capture cross sections $\sigma_{\text {cap }}$ and rate constants $k_{\text {cap }}$ are easily calculated. We again represent the results as scaled quantities ${ }^{3}$. The scaled energy-dependent rate coefficients. 
i.e. the ratio of the capture rate coefficients and the energy-independent Langevin rate coefficient, as a function of $\kappa$ reads $^{3}$

$$
\chi(\kappa)=\sum_{l} \chi_{l}(\kappa)=\sum_{l} \frac{(2 l+1)}{2 \kappa} P_{l}(\kappa)
$$

The energy-dependent rate coefficients $k_{\text {cap }}(E)$ in conventional units are recovered from $\chi(\kappa)$ and the Langevin rate constant $k_{L}$ from eqn (1.5) through

$$
k_{\text {cap }}(E)=\chi(\kappa) k_{L}
$$

with $\varepsilon=E / E_{s}=\kappa^{2} / 2$. Finally, the capture cross section is calculated from $k_{\text {cap }}(E)$ by the standard relation

$$
\sigma_{\text {cap }}(E)=k_{\text {cap }}(E) / \sqrt{2 E / \mu}
$$

which in scaled form corresponds to

$$
\sigma_{\text {red }}=\sigma_{\text {cap }} / 2 \pi R_{\mathrm{s}}^{2}=\chi(\kappa) / \kappa
$$

The thermally averaged counterpart of $\chi(\kappa), \bar{\chi}$, depends on the reduced temperature $\theta=k_{\mathrm{B}} T \mu R_{s}^{2} / \hbar^{2}=k_{\mathrm{B}} T / E_{s}$ and is expressed as

$$
\begin{aligned}
\bar{\chi}(\theta) & =\int_{0}^{\infty} \chi(\kappa) F(\kappa) \mathrm{d} \kappa \\
F(\kappa) & =\frac{2 \theta^{-3 / 2}}{(2 \pi)^{1 / 2}} \kappa^{2} \exp \left(-\frac{\kappa^{2}}{2 \theta}\right)
\end{aligned}
$$

Similar to eqn (3.2) one then has

$$
k_{\text {cap }}(T)=\chi(\theta) k_{L}
$$

with $T=\theta E_{s} / k_{\mathrm{B}}$. In the following we demonstrate how fast capture cross sections and capture rate constants are approached upon summation over a limited number of partial waves $l$. We employ scaled quantities and we compare results for zero-size and finite-size targets.

Fig. 5 compares scaled capture cross sections $\sigma_{\text {red }}(\varepsilon)$ as a function of the scaled energy $\varepsilon=\kappa^{2} / 2$ for $\rho_{0}=0$ and 1.0 . The summations over partial waves, for illustration of the contributions of individual partial waves, extend over $l=0$, $0 \rightarrow 1$, and $0 \rightarrow 2$ for $\rho_{0}=0$, whereas $l=0,0 \rightarrow 1,0 \rightarrow 2$, $0 \rightarrow 3$, and $0 \rightarrow 4$ are shown for $\rho_{0}=1$. In both cases also the all-wave results are shown. One observes that finite-size effects become apparent at $\varepsilon$ above about 0.1 . Furthermore, one also notices that quantum effects are most important for $\varepsilon$ values below a similar magnitude. $\sigma_{\text {red }}$ in the quantum range then increases to values above its classical value

$$
\sigma_{\text {red }}^{\text {cl }}(\varepsilon)=\rho_{0}{ }^{2} / 2+(2 \varepsilon)^{1 / 2}
$$

which, for $\rho_{0}=0$, is also shown in Fig. 5. One realizes that, at $\varepsilon$ smaller than unity, the summation over only few partial waves suffices to bring $\sigma_{\text {red }}$ close to $\sigma_{\text {red }}^{\text {cl }}$. The finite-size capture probabilities from section 2 apparently are precise enough to provide convergence to the correct classical limit.

We have illustrated in section 2 that the iM approach does not provide reliable results for transmission coefficients in the tunnelling range, i.e. for $P_{l}\left(\kappa, \rho_{0}\right)$ below about 0.1 . For this range, the present iM approach can only be considered as a

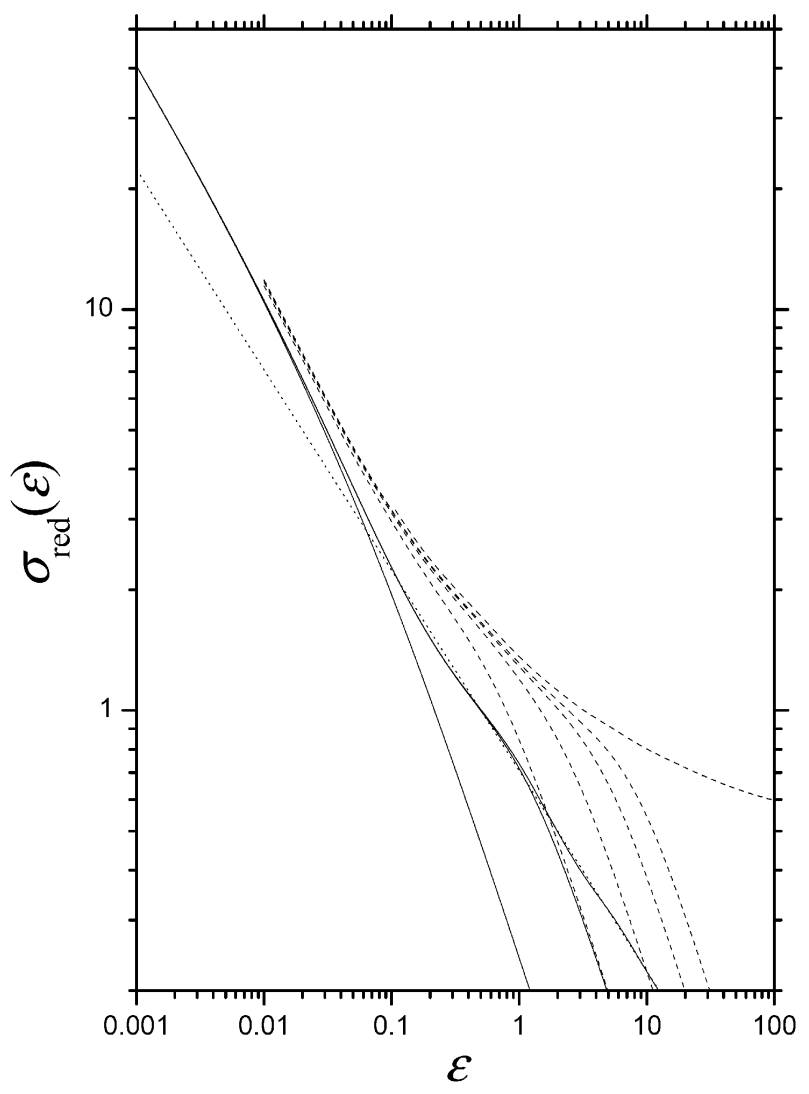

Fig. 5 Scaled capture cross sections $\sigma_{\text {red }}$ as a function of the scaled energy $\varepsilon$ (full lines: $\rho_{0}=0$, lines from left to right: $l=0, l=0 \rightarrow 1$, all $l$; dashed lines: $\rho_{0}=1$, lines from left to right: $l=0 \rightarrow 1, l=0 \rightarrow 2$, $l=0 \rightarrow 3, l=0 \rightarrow 4$, all $l$; dotted line: classical result for $\rho_{0}=0$ ).

first step towards a solution of the problem. For this reason, the cross sections obtained with the iM approach cannot be trusted at $\varepsilon<0.01$.

Fig. 6 illustrates the finite-size effects in scaled thermal capture rate constants and compares the results with the scaled classical rate constants corresponding to eqn (1.4), i.e.

$$
\bar{\chi}^{\mathrm{cl}}(\theta)=\rho_{0}^{2}(\theta / 2)^{1 / 2}+1
$$

Two observations appear worth noticing. At small temperatures, $\theta \ll 1$, with dominant s-wave capture, the Bethe quantum limit of $\bar{\chi}(\theta \rightarrow 0)$, which is equal to 2 for $\rho_{0}=0$, is not approached by the classical eqn (3.9) for which $\bar{\chi}^{\mathrm{cl}}(\theta \rightarrow 0)=1$. With increasing $\theta$, an increasing number of partial waves contributes where the size effects get stronger with increasing $l$, until the classical eqn (3.9) is approached. Because of the counteraction of two effects, there is a rate constant minimum at values of $\theta$ between 0.1 and 1. Fig. 6 also includes a comparison between accurate results for zero-size targets and results obtained by the iM approach. It appears that the latter is good enough for most practical applications.

\section{Conclusions}

A series of conclusions can be drawn from the derived results. Finite-size effects decrease with decreasing $l$ and the trends observed for higher partial waves suggest that such effects can 


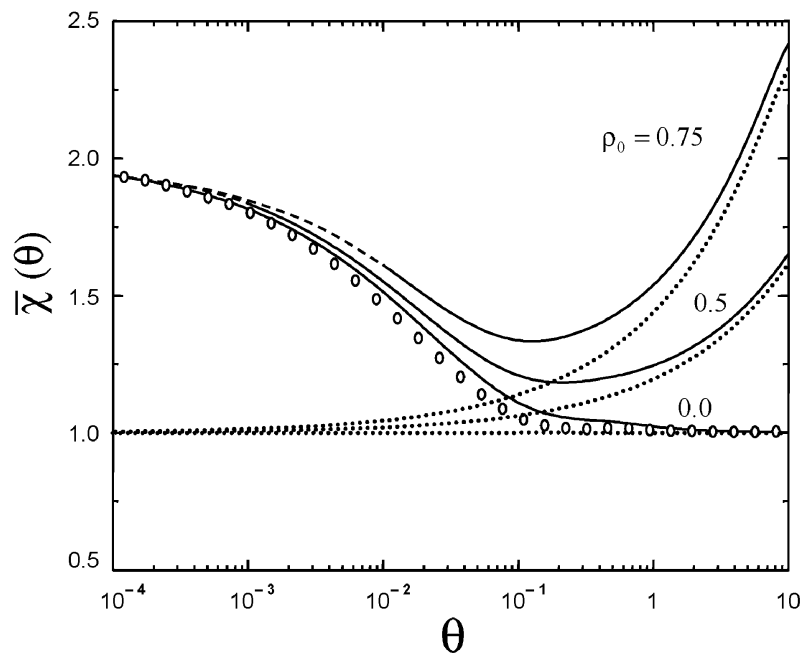

Fig. 6 Scaled thermal capture rate coefficients $\bar{\chi}(\theta)$ from eqn (3.7) as a function of the scaled temperature, see eqn (3.6), for zero-size $\left(\rho_{0}=0\right)$ and finite-size ( $\rho_{0}=0.5$ and 0.75$)$ targets (dotted lines: classical results from eqn (3.9), full lines: iM results, see text; open circles: accurate results for $\rho_{0}=0$ from ref. 3).

be neglected for s-waves. The shift relationships of eqn (2.18)-(2.21) allow one to express the finite-size effects for partial-wave selected capture probabilities in terms of the analytical expressions for zero-size targets derived in ref. 15. One may ask where such finite-size effects may become of practice relevance. On the one hand, large targets generally will be characterized by contributions from so many partial waves that the classical capture cross sections from eqn (1.3) will provide a sufficient description. On the other hand, targets with decreasing size will generally be dominated by a decreasing number of partial waves with decreasing $l$. For these, however, the importance of finite-size effects will decrease. There should, therefore, only be a limited range of target sizes and a limited range of intermediate energies and/or temperatures where partial-wave finite-size effects become of relevance. Such intermediate ranges have been illustrated in section 3 . In a specific application, we found ${ }^{13}$ that electron capture by $\mathrm{C}_{60}$ at energies $E$ around $0.1 \mathrm{eV}$ shows some deviations from the classical relationship of eqn (1.4). However, these deviations are still small. Electron capture by $\mathrm{SF}_{6}$, on the other hand, is dominated by s-wave capture where finite-size effects are probably negligible. Only for target sizes somewhere between these two cases might quantum partial-wave finite-size effects become noticeable in experiments.

\section{Acknowledgements}

Helpful discussions of this work with A. A. Viggiano, as well as financial support by the EOARD Grant Award No. FA 8655-09-1-3001 are gratefully acknowledged. Particular thanks go to I. Litvin who extended the Table 1 for $l=1-4$ from ref. 14 and 15 up to $l=12$. Technical help by him and A. I. Maergoiz is also gratefully acknowledged.

\section{References}

1 E. Vogt and G. H. Wannier, Phys. Rev., 1954, 95, 1190.

2 I. I. Fabrikant and H. Hotop, Phys. Rev. A: At., Mol., Opt. Phys., 2001, 63, 022706.

3 E. I. Dashevskaya, A. I. Maergoiz, J. Troe, I. Litvin and E. E. Nikitin, J. Chem. Phys., 2003, 118, 7313.

4 H. Hotop, M.-W. Ruf, M. Allan and I. I. Fabrikant, Adv. At. Mol. Opt. Phys., 2003, 49, 85.

5 J. Troe, T. M. Miller and A. A. Viggiano, J. Chem. Phys., 2007, 127, 244303.

6 L. D. Landau, E. M. Lifshitz and L. P. Pitaevskii, Electrodynamics of Continuous Media, Pergamon Press, Oxford, 2nd edn, 1984.

7 C. E. Klots, J. Chem. Phys., 1994, 100, 1035.

8 E. E. B. Campbell and R. D. Levine, Annu. Rev. Phys. Chem., 2000, 51, 65

9 J. U. Andersen, E. Bonderup and K. Hansen, J. Phys. B: At., Mol. Opt. Phys., 2002, 35, R1.

10 V. Kasperovich, K. Wong, G. Tikhonov and V. V. Kresin, Phys. Rev. Lett., 2000, 85, 2729.

11 M. Lezius, Int. J. Mass Spectrom., 2003, 223-224, 447.

12 H. Yan, S. Yu, X. Wang, Y. He, W. Huang and M. Yang, Chem. Phys. Lett., 2008, 456, 223.

13 A. A. Viggiano, T. M. Miller, J. F. Friedman, L. C. Schaffer, N. S. Shuman and J. Troe, J. Chem. Phys., 2010, 132, 194307.

14 E. I. Dashevskaya, I. Litvin, E. E. Nikitin and J. Troe, Phys. Chem. Chem. Phys., 2008, 10, 1270.

15 E. I. Dashevskaya, I. Litvin, E. E. Nikitin and J. Troe, Phys. Chem. Chem. Phys., 2009, 11, 9364.

16 A. S. Dickinson, Mol. Phys., 1970, 18, 441.

17 B. Lundborg, Math. Proc. Cambridge Philos. Soc., 1977, 81, 463.

18 R. P. Bell, J. Chem. Soc., Faraday Trans. 2, 1978, 74, 688.

19 Z. Ahmed, Phys. Lett. A, 1991, 157, 1.

20 Z. Ahmed, Phys. Rev. A: At., Mol., Opt. Phys., 1993, 47, 4761. 